\section{Crystal structure of $N^{\prime}-[(E)$-(4-chloro- phenyl)(phenyl)methylidene]-4-methyl- benzenesulfonohydrazide}

\author{
J. Balaji, ${ }^{a}$ S. Prabu, ${ }^{a}$ J. J. F. Xavier ${ }^{b}$ and P. Srinivasan ${ }^{a *}$ \\ a Department of Physics, UCEP, Panruti 607 106, TamilNadu, India, and \\ bepartment of Chemistry, UCEP, Panruti 607 106, TamilNadu, India. *Correspon- \\ dence e-mail: sril35@gmail.com
}

Received 16 October 2014; accepted 5 December 2014

Edited by G. Smith, Queensland University of Technology, Australia

The title compound, $\mathrm{C}_{20} \mathrm{H}_{17} \mathrm{ClN}_{2} \mathrm{O}_{2} \mathrm{~S}$, was obtained by a condensation reaction between 4-chlorobenzophenone and tosyl hydrazide. The plane of the methyl-substituted benzene ring forms dihedral angles of 20.12 (12) and $78.43(13)^{\circ}$ with those of the chlorine-substituted benzene ring and the benzene ring, respectively, with the last two rings forming a dihedral angle of $67.81(13)^{\circ}$. The chlorine substituent was also found to be 0.868 (2):0.132 (2) disordered over these two rings. In the crystal, molecules are linked through pairs of $\mathrm{N}-$ $\mathrm{H}$... O hydrogen bonds, giving centrosymmetric cyclic dimers [graph set $R_{2}^{2}(8)$ ], which are linked by weak $\mathrm{C}-\mathrm{H} \cdots \mathrm{O}$ and $\mathrm{C}-\mathrm{H} \cdots \mathrm{Cl}$ interactions into a chain structure which extends along the $a$-axis direction.

Keywords: crystal structure; benzenesulfonohydrazide; hydrogen bonding; condensation reaction; centrosymmetric dimers.

CCDC reference: 1037752

\section{Related literature}

Benzophenone and its derivatives have been investigated extensively for their biological activities such as anti-fungal and anti-inflammatory, see: Khanum et al. (2004). For similar structures, see: Ajani et al. (2010); Gerdemann et al. (2002); Kutzke et al. (2000); Shen et al. (2012); Zhang (2011).<smiles>Cc1ccc(S(=O)(=O)N/N=C(/c2ccccc2)c2ccc(Cl)cc2)cc1</smiles>

\section{Experimental}

\subsection{Crystal data}

$$
\begin{aligned}
& \mathrm{C}_{20} \mathrm{H}_{17} \mathrm{ClN}_{2} \mathrm{O}_{2} \mathrm{~S} \\
& M_{r}=384.87 \\
& \text { Monoclinic, } P 2_{1} / c \\
& a=12.6808(6) \AA \\
& b=9.3857(5) \AA \\
& c=16.3974(7) \AA \\
& \beta=106.187(2)^{\circ}
\end{aligned}
$$

$$
\begin{aligned}
& V=1874.22(16) \AA^{3} \\
& Z=4 \\
& \text { Mo } K \alpha \text { radiation } \\
& \mu=0.33 \mathrm{~mm}^{-1} \\
& T=293 \mathrm{~K} \\
& 0.35 \times 0.30 \times 0.25 \mathrm{~mm}
\end{aligned}
$$

\subsection{Data collection}

Bruker Kappa APEXII CCD diffractometer

Absorption correction: multi-scan (SADABS; Sheldrick, 1996) $T_{\min }=0.891, T_{\max }=0.930$

21401 measured reflections 3300 independent reflections 2416 reflections with $I>2 \sigma(I)$ $R_{\text {int }}=0.032$

\subsection{Refinement}

$R\left[F^{2}>2 \sigma\left(F^{2}\right)\right]=0.039$

$w R\left(F^{2}\right)=0.105$

$S=1.06$

3300 reflections

253 parameters

4 restraints
$\mathrm{H}$ atoms treated by a mixture of independent and constrained refinement $\Delta \rho_{\max }=0.35$ e $\AA^{-3}$ $\Delta \rho_{\min }=-0.33 \mathrm{e}^{-3}$

Table 1

Hydrogen-bond geometry $\left(\AA{ }^{\circ}\right)$.

\begin{tabular}{lllll}
\hline$D-\mathrm{H} \cdots A$ & $D-\mathrm{H}$ & $\mathrm{H} \cdots A$ & $D \cdots A$ & $D-\mathrm{H} \cdots A$ \\
\hline $\mathrm{N} 1-\mathrm{H} 1 A \cdots \mathrm{O} 2^{\mathrm{i}}$ & $0.88(2)$ & $2.19(2)$ & $3.024(3)$ & $160(2)$ \\
$\mathrm{C} 10-\mathrm{H} 10 \cdots \mathrm{Cl} 1^{\prime \text { ii }}$ & 0.93 & 2.76 & $3.476(7)$ & 134 \\
$\mathrm{C} 16-\mathrm{H} 16 \cdots \mathrm{O} 1^{\text {iii }}$ & 0.93 & 2.54 & $3.339(3)$ & 145 \\
\hline
\end{tabular}

Symmetry codes: (i) $-x+1,-y+2,-z+1 ; \quad$ (ii) $\quad-x+2, y+\frac{1}{2},-z+\frac{3}{2} ; \quad$ (iii) $-x+2,-y+2,-z+1$.

Data collection: $A P E X 2$ (Bruker, 2004); cell refinement: $A P E X 2$ and SAINT (Bruker, 2004); data reduction: SAINT and XPREP (Bruker, 2004); $\operatorname{program}(\mathrm{s})$ used to solve structure: SIR92 (Altomare et al., 1993); program(s) used to refine structure: SHELXL97 (Sheldrick, 2008); molecular graphics: ORTEP-3 for Windows (Farrugia, 2012) and Mercury (Macrae et al., 2008); software used to prepare material for publication: SHELXL97. 


\section{Acknowledgements}

JB thanks the CTDT, Anna University, for funding the project (AU/ROT/BIT/R\&D/YFP/PAN/PHY/2013-14/001). The authors thank the SAIF, IITM, Madras, for helping with the XRD studies.

Supporting information for this paper is available from the IUCr electronic archives (Reference: ZS2319).

\section{References}

Ajani, O. O., Obafemi, C. A., Nwinyi, O. C. \& Akinpelu, D. A. (2010). Bioorg. Med. Chem. 18, 214-221.
Altomare, A., Cascarano, G., Giacovazzo, C. \& Guagliardi, A. (1993). J. Appl. Cryst. 26, 343-350.

Bruker (2004). APEX2, SAINT and XPREP. Bruker AXS Inc., Madison, Wisconsin, USA.

Farrugia, L. J. (2012). J. Appl. Cryst. 45, 849-854.

Gerdemann, C., Eicken, C. \& Krebs, B. (2002). Acc. Chem. Res. 35, 183-191.

Khanum, S. A., Venu, T. D., Shashikanth, S. \& Firdouse, A. (2004). Bioorg. Med. Chem. Lett. 12, 2093-2095.

Kutzke, H., Klapper, H., Hammond, R. B. \& Roberts, K. J. (2000). Acta Cryst. B56, 486-496.

Macrae, C. F., Bruno, I. J., Chisholm, J. A., Edgington, P. R., McCabe, P., Pidcock, E., Rodriguez-Monge, L., Taylor, R., van de Streek, J. \& Wood, P. A. (2008). J. Appl. Cryst. 41, 466-470.

Sheldrick, G. M. (1996). SADABS. University of Göttingen, Germany.

Sheldrick, G. M. (2008). Acta Cryst. A64, 112-122.

Shen, X.-H., Zhu, L.-X., Shao, L.-J. \& Zhu, Z.-F. (2012). Acta Cryst. E68, o297.

Zhang, W.-G. (2011). Acta Cryst. E67, o233. 


\section{supporting information}

Acta Cryst. (2015). E71, o45-o46 [https://doi.org/10.1107/S2056989014026723]

\section{Crystal structure of $N^{\prime}-[(E)-(4-c h l o r o p h e n y l)(p h e n y l)$ methylidene]-4-methyl- benzenesulfonohydrazide}

\section{J. Balaji, S. Prabu, J. J. F. Xavier and P. Srinivasan}

\section{S1. Chemical context}

Currently the hydrazones have attracted considerable attention due to their biological activities and a number of crystal structures of these compounds have been reported (Ajani et al., 2010); Gerdemann et al., 2002; Kutzke et al., 2000); Zhang, 2011; Shen et al., 2012). Benzophenone and its derivatives have also been extensively investigated for their biological activities such as anti-fungal and anti-inflammatory (Khanum et al., 2004). In view of the importance of these analogs, the title compound, $\mathrm{C}_{20} \mathrm{H}_{17} \mathrm{ClN}_{2} \mathrm{O}_{2} \mathrm{~S}$, was synthesized in a Schiff base condensation reaction between 4-chlorobenzophenone and tosyl hydrazide and its structure is reported herein.

In this compound (Fig. 1) the benzene ring (C1-C6) forms dihedral angles of $20.12(12)$ and $78.43(13)^{\circ}$ with the chlorine-substituted benzene ring $(\mathrm{C} 8-\mathrm{C} 13)$ and the benzene ring $(\mathrm{C} 14-\mathrm{C} 19)$, respectively. The molecule is twisted, with the dihedral angle between the two benzene rings (C8-C13 and $\mathrm{C} 14-\mathrm{C} 19)$ of the parent moiety being $67.81(13)^{\circ}$. In the crystal, molecules are linked through intermolecular $\mathrm{N} 1-\mathrm{H}^{\cdots} \cdot \mathrm{O} 2^{\mathrm{i}}$ hydrogen-bond pairs (Table 1) giving centrosymmetric cyclic dimers [graph set $R_{2}^{2}(8)$ ] which are linked by weak $\mathrm{C}-\mathrm{H} \cdots \mathrm{O}$ and $\mathrm{C}-\mathrm{H} \cdots \mathrm{Cl}$ interactions into a chain structure which extends along $a$ (Fig. 2).

\section{S2. Synthesis and crystallization}

4-Chlorobenzophenone $(0.15 \mathrm{~g}, 1 \mathrm{mmol})$ and tosyl hydrazide $(0.186 \mathrm{~g}, 1 \mathrm{mmol})$ were dissolved in ethanol $(50 \mathrm{ml})$. The reaction mixture was heated under reflux for $3 \mathrm{hr}$ and cooled gradually to room temperature. Crystals suitable for X-ray diffraction analysis were obtained by slow room temperature evaporation of the solution containing the compound.

\section{S3. Refinement}

All $\mathrm{H}$ atoms were positioned geometrically and treated as riding on their parent atoms with $\mathrm{C}-\mathrm{H}=0.93 \AA$ (aromatic) or $0.96 \AA$ (methyl) and $\mathrm{N} 1-\mathrm{H}=0.89 \pm 2 \AA$ with $U_{\text {iso }}(\mathrm{H})=1.2 U_{\text {eq }}\left(\mathrm{N}, \mathrm{C}_{\text {aromatic }}\right)$ or $1.5 U_{\text {eq }}\left(\mathrm{C}_{\text {methyl }}\right)$. The chlorine substituent was also found to be disordered over the $\mathrm{C} 8-\mathrm{C} 13(\mathrm{Cl1})$ and $\mathrm{C} 14-\mathrm{C} 19\left(\mathrm{Cl1} 1^{\prime}\right)$ rings of the original benzophenone moiety [occupancy factors $0.868(2): 0.132(2)$, respectively]. 


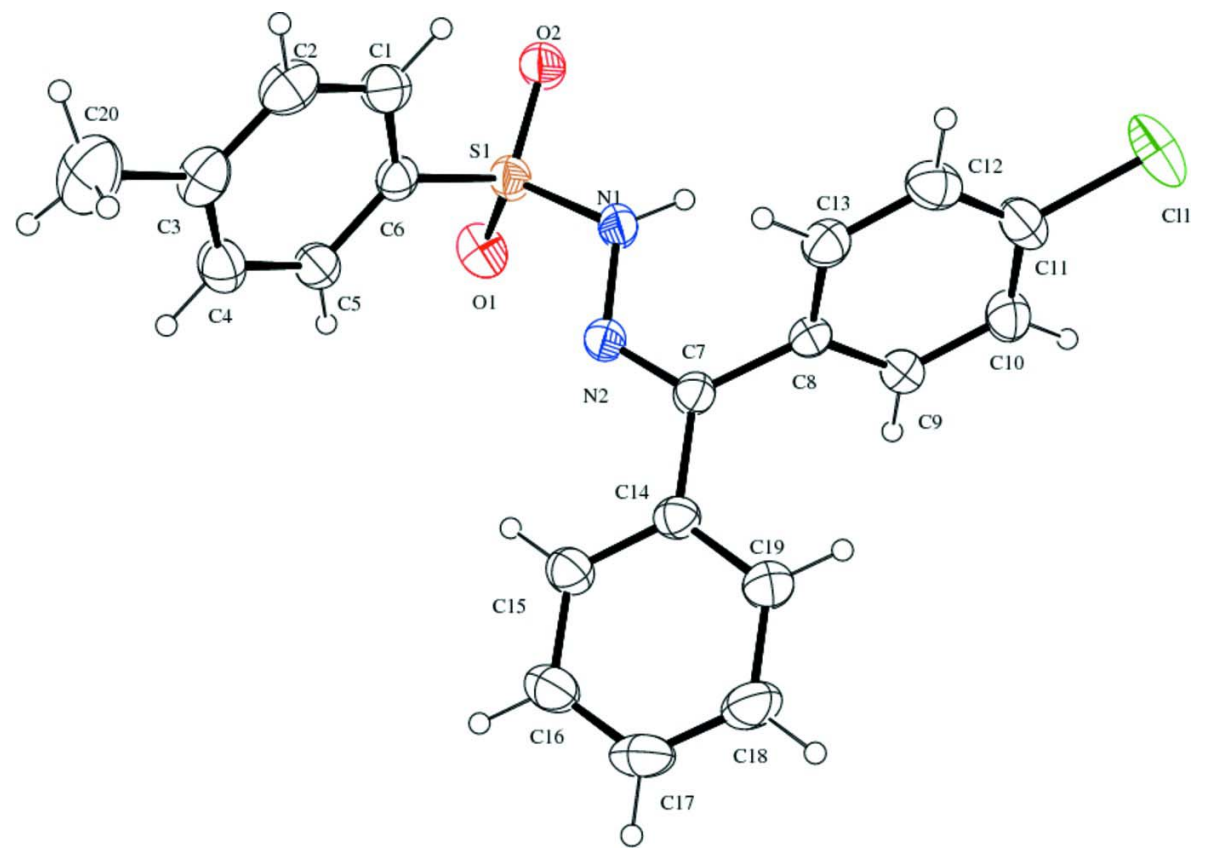

Figure 1

The molecular structure of the title compound showing the atom labelling scheme. The displacement ellipsoids are drawn at the $30 \%$ probability level

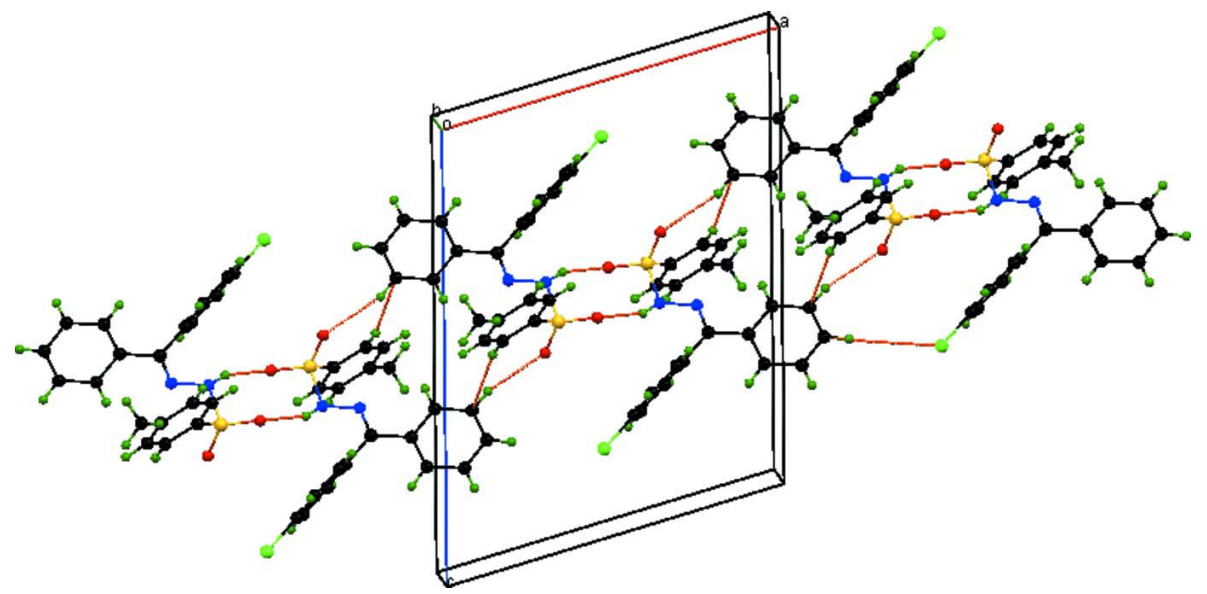

Figure 2

A view of the crystal packing of the title compound. The various hydrogen bonds are indicated by dashed lines (see Table 1 for details).

$N^{\prime}$-[(E)-(4-Chlorophenyl)(phenyl)methylidene]-4-methylbenzenesulfonohydrazide

\section{Crystal data}

$\mathrm{C}_{20} \mathrm{H}_{17} \mathrm{ClN}_{2} \mathrm{O}_{2} \mathrm{~S}$

$M_{r}=384.87$

Monoclinic, $P 2{ }_{1} / c$

Hall symbol: -P 2ybc

$a=12.6808$ (6) $\AA$

$b=9.3857$ (5) $\AA$

$$
\begin{aligned}
& c=16.3974(7) \AA \\
& \beta=106.187(2)^{\circ} \\
& V=1874.22(16) \AA^{3} \\
& Z=4 \\
& F(000)=800 \\
& D_{\mathrm{x}}=1.364 \mathrm{Mg} \mathrm{m}^{-3}
\end{aligned}
$$


Mo $K \alpha$ radiation, $\lambda=0.71073 \AA$

Cell parameters from 6873 reflections

$\theta=2.5-25.2^{\circ}$

$\mu=0.33 \mathrm{~mm}^{-1}$

\section{Data collection}

Bruker Kappa APEXII CCD

diffractometer

Radiation source: fine-focus sealed tube

Graphite monochromator

$\omega$ and $\varphi$ scans

Absorption correction: multi-scan

(SADABS; Sheldrick, 1996)

$T_{\min }=0.891, T_{\max }=0.930$

\section{Refinement}

Refinement on $F^{2}$

Least-squares matrix: full

$R\left[F^{2}>2 \sigma\left(F^{2}\right)\right]=0.039$

$w R\left(F^{2}\right)=0.105$

$S=1.06$

3300 reflections

253 parameters

4 restraints

Primary atom site location: structure-invariant direct methods

Secondary atom site location: difference Fourier map
$T=293 \mathrm{~K}$

Block, brown

$0.35 \times 0.30 \times 0.25 \mathrm{~mm}$

21401 measured reflections

3300 independent reflections

2416 reflections with $I>2 \sigma(I)$

$R_{\text {int }}=0.032$

$\theta_{\max }=25.0^{\circ}, \theta_{\min }=2.5^{\circ}$

$h=-15 \rightarrow 15$

$k=-11 \rightarrow 11$

$l=-18 \rightarrow 19$

Hydrogen site location: inferred from neighbouring sites

$\mathrm{H}$ atoms treated by a mixture of independent and constrained refinement

$w=1 /\left[\sigma^{2}\left(F_{\mathrm{o}}^{2}\right)+(0.0351 P)^{2}+1.1726 P\right]$

where $P=\left(F_{\mathrm{o}}^{2}+2 F_{\mathrm{c}}^{2}\right) / 3$

$(\Delta / \sigma)_{\max }=0.001$

$\Delta \rho_{\max }=0.35$ e $\AA^{-3}$

$\Delta \rho_{\min }=-0.33$ e $\AA^{-3}$

Extinction correction: SHELXL97 (Sheldrick, 2008), $\mathrm{Fc}^{*}=\mathrm{kFc}\left[1+0.001 \mathrm{xFc}^{2} \lambda^{3} / \sin (2 \theta)\right]^{-1 / 4}$

Extinction coefficient: 0.0025 (7)

Special details

Geometry. All e.s.d.'s (except the e.s.d. in the dihedral angle between two 1.s. planes) are estimated using the full covariance matrix. The cell e.s.d.'s are taken into account individually in the estimation of e.s.d.'s in distances, angles and torsion angles; correlations between e.s.d.'s in cell parameters are only used when they are defined by crystal symmetry. An approximate (isotropic) treatment of cell e.s.d.'s is used for estimating e.s.d.'s involving l.s. planes.

Refinement. Refinement of $F^{2}$ against ALL reflections. The weighted $R$-factor $w R$ and goodness of fit $S$ are based on $F^{2}$, conventional $R$-factors $R$ are based on $F$, with $F$ set to zero for negative $F^{2}$. The threshold expression of $F^{2}>\sigma\left(F^{2}\right)$ is used only for calculating $R$-factors (gt) etc. and is not relevant to the choice of reflections for refinement. $R$-factors based on $F^{2}$ are statistically about twice as large as those based on $F$, and $R$ - factors based on ALL data will be even larger.

Fractional atomic coordinates and isotropic or equivalent isotropic displacement parameters $\left(\AA^{2}\right)$

\begin{tabular}{llllll}
\hline & $x$ & $y$ & $z$ & $U_{\text {iso }} * / U_{\text {eq }}$ & Occ. $(<1)$ \\
\hline C1 & $0.6473(2)$ & $0.5896(3)$ & $0.50383(16)$ & $0.0567(7)$ & \\
H1 & 0.5830 & 0.6014 & 0.5198 & $0.068^{*}$ & \\
C2 & $0.6980(3)$ & $0.4588(3)$ & $0.51169(18)$ & $0.0650(8)$ & \\
H2 & 0.6676 & 0.3825 & 0.5334 & $0.078^{*}$ & \\
C3 & $0.7928(3)$ & $0.4383(3)$ & $0.48812(18)$ & $0.0632(7)$ & \\
C4 & $0.8369(2)$ & $0.5531(3)$ & $0.45737(17)$ & $0.0612(7)$ & \\
H4 & 0.9013 & 0.5410 & 0.4416 & $0.073^{*}$ & \\
C5 & $0.7885(2)$ & $0.6855(3)$ & $0.44933(16)$ & $0.0510(6)$ & \\
H5 & 0.8200 & 0.7621 & 0.4288 & $0.061^{*}$ & \\
C6 & $0.69263(19)$ & $0.7028(3)$ & $0.47211(14)$ & $0.0445(6)$ & \\
C7 & $0.81124(18)$ & $1.0259(2)$ & $0.65813(15)$ & $0.0430(6)$ &
\end{tabular}




\begin{tabular}{|c|c|c|c|c|c|}
\hline $\mathrm{C} 8$ & $0.73683(18)$ & $1.0797(3)$ & $0.70683(14)$ & $0.0429(6)$ & \\
\hline C9 & $0.7223(2)$ & $1.2238(3)$ & $0.71776(16)$ & $0.0511(6)$ & \\
\hline H9 & 0.7612 & 1.2898 & 0.6953 & $0.061^{*}$ & \\
\hline $\mathrm{C} 10$ & $0.6505(2)$ & $1.2706(3)$ & $0.76179(17)$ & $0.0581(7)$ & \\
\hline $\mathrm{H} 10$ & 0.6405 & 1.3675 & 0.7691 & $0.070 *$ & \\
\hline $\mathrm{C} 11$ & $0.5943(2)$ & $1.1708(3)$ & $0.79449(16)$ & $0.0587(7)$ & \\
\hline $\mathrm{C} 12$ & $0.6081(2)$ & $1.0282(3)$ & $0.78491(17)$ & $0.0605(7)$ & \\
\hline $\mathrm{H} 12$ & 0.5692 & 0.9624 & 0.8077 & $0.073 *$ & \\
\hline $\mathrm{C} 13$ & $0.6794(2)$ & $0.9831(3)$ & $0.74156(15)$ & $0.0525(6)$ & \\
\hline H13 & 0.6895 & 0.8859 & 0.7353 & $0.063 *$ & \\
\hline $\mathrm{C} 14$ & $0.93096(18)$ & $1.0480(3)$ & $0.68959(15)$ & $0.0453(6)$ & \\
\hline C15 & $0.9995(2)$ & $1.0041(3)$ & $0.64206(17)$ & $0.0541(7)$ & \\
\hline H15 & 0.9696 & 0.9608 & 0.5897 & $0.065^{*}$ & \\
\hline C16 & $1.1112(2)$ & $1.0234(3)$ & $0.6711(2)$ & $0.0632(8)$ & \\
\hline H16 & 1.1566 & 0.9927 & 0.6388 & $0.076^{*}$ & \\
\hline C17 & $1.1549(2)$ & $1.0880(3)$ & $0.7477(2)$ & $0.0699(9)$ & \\
\hline H17 & $1.2283(17)$ & $1.100(4)$ & $0.769(2)$ & $0.084^{*}$ & \\
\hline $\mathrm{C} 18$ & $1.0896(2)$ & $1.1329(3)$ & $0.7961(2)$ & $0.0723(8)$ & \\
\hline $\mathrm{H} 18$ & 1.1203 & 1.1770 & 0.8481 & $0.087^{*}$ & \\
\hline C19 & $0.9777(2)$ & $1.1124(3)$ & $0.76732(17)$ & $0.0610(7)$ & \\
\hline H19 & 0.9331 & 1.1421 & 0.8005 & $0.073^{*}$ & \\
\hline $\mathrm{C} 20$ & $0.8462(3)$ & $0.2933(4)$ & 0.4951 & $0.1014(12)$ & \\
\hline $\mathrm{H} 20 \mathrm{~A}$ & 0.8929 & 0.2890 & 0.4580 & $0.152^{*}$ & \\
\hline $\mathrm{H} 20 \mathrm{~B}$ & 0.7905 & 0.2213 & 0.4791 & $0.152 *$ & \\
\hline $\mathrm{H} 20 \mathrm{C}$ & 0.8893 & 0.2776 & 0.5526 & $0.152^{*}$ & \\
\hline N1 & $0.66524(16)$ & $0.9315(2)$ & $0.55792(13)$ & $0.0548(6)$ & \\
\hline N2 & $0.77696(15)$ & $0.9565(2)$ & $0.58850(13)$ & $0.0498(5)$ & \\
\hline $\mathrm{O} 1$ & $0.67110(15)$ & $0.9520(2)$ & $0.40485(12)$ & $0.0639(5)$ & \\
\hline $\mathrm{O} 2$ & $0.51226(13)$ & $0.8526(2)$ & $0.44334(11)$ & $0.0599(5)$ & \\
\hline $\mathrm{S} 1$ & $0.62845(5)$ & $0.86959(7)$ & $0.46061(4)$ & $0.0477(2)$ & \\
\hline $\mathrm{Cl1}$ & $0.50538(8)$ & $1.22428(12)$ & $0.85037(6)$ & $0.0880(4)$ & $0.868(2)$ \\
\hline $\mathrm{Cl1}^{\prime}$ & $1.2814(4)$ & $1.1131(6)$ & $0.7865(4)$ & 0.0616 (19) & $0.132(2)$ \\
\hline $\mathrm{H} 1 \mathrm{~A}$ & $0.6174(19)$ & $0.988(2)$ & $0.5711(16)$ & $0.061(8)^{*}$ & \\
\hline
\end{tabular}

Atomic displacement parameters $\left(\AA^{2}\right)$

\begin{tabular}{lllllll}
\hline & $U^{11}$ & $U^{22}$ & $U^{33}$ & $U^{12}$ & $U^{13}$ & $U^{23}$ \\
\hline C1 & $0.0582(16)$ & $0.0650(18)$ & $0.0486(15)$ & $-0.0090(14)$ & $0.0175(12)$ & $-0.0045(13)$ \\
C2 & $0.078(2)$ & $0.0537(18)$ & $0.0595(17)$ & $-0.0106(15)$ & $0.0136(15)$ & $0.0068(14)$ \\
C3 & $0.073(2)$ & $0.0506(17)$ & $0.0568(17)$ & $0.0071(14)$ & $0.0038(14)$ & $0.0010(14)$ \\
C4 & $0.0578(17)$ & $0.0611(18)$ & $0.0649(18)$ & $0.0115(14)$ & $0.0173(14)$ & $-0.0015(14)$ \\
C5 & $0.0493(15)$ & $0.0498(15)$ & $0.0558(16)$ & $0.0009(12)$ & $0.0179(12)$ & $0.0001(12)$ \\
C6 & $0.0467(14)$ & $0.0476(14)$ & $0.0371(13)$ & $-0.0008(11)$ & $0.0081(11)$ & $-0.0058(11)$ \\
C7 & $0.0414(13)$ & $0.0402(13)$ & $0.0454(14)$ & $0.0014(10)$ & $0.0087(11)$ & $-0.0039(11)$ \\
C8 & $0.0381(12)$ & $0.0476(14)$ & $0.0408(13)$ & $-0.0034(10)$ & $0.0072(10)$ & $-0.0069(11)$ \\
C9 & $0.0473(14)$ & $0.0517(15)$ & $0.0577(16)$ & $-0.0038(12)$ & $0.0200(12)$ & $-0.0072(12)$ \\
C10 & $0.0584(16)$ & $0.0554(16)$ & $0.0617(17)$ & $0.0062(13)$ & $0.0191(14)$ & $-0.0084(14)$ \\
C11 & $0.0449(15)$ & $0.085(2)$ & $0.0483(15)$ & $0.0102(14)$ & $0.0167(12)$ & $0.0013(15)$
\end{tabular}


supporting information

\begin{tabular}{lllllll}
\hline C12 & $0.0563(16)$ & $0.075(2)$ & $0.0517(16)$ & $-0.0085(15)$ & $0.0174(13)$ & $0.0071(14)$ \\
C13 & $0.0557(15)$ & $0.0526(15)$ & $0.0478(15)$ & $-0.0043(12)$ & $0.0122(12)$ & $-0.0031(12)$ \\
C14 & $0.0399(13)$ & $0.0422(13)$ & $0.0510(15)$ & $0.0005(10)$ & $0.0081(11)$ & $0.0014(11)$ \\
C15 & $0.0471(14)$ & $0.0533(16)$ & $0.0627(17)$ & $0.0036(12)$ & $0.0165(13)$ & $0.0001(13)$ \\
C16 & $0.0457(15)$ & $0.0585(17)$ & $0.088(2)$ & $0.0035(13)$ & $0.0225(15)$ & $0.0049(16)$ \\
C17 & $0.0399(15)$ & $0.0563(18)$ & $0.104(3)$ & $-0.0040(14)$ & $0.0041(17)$ & $0.0068(17)$ \\
C18 & $0.0513(17)$ & $0.073(2)$ & $0.078(2)$ & $-0.0055(15)$ & $-0.0058(15)$ & $-0.0135(17)$ \\
C19 & $0.0481(15)$ & $0.0690(18)$ & $0.0612(17)$ & $-0.0013(13)$ & $0.0076(13)$ & $-0.0123(15)$ \\
C20 & $0.122(3)$ & $0.059(2)$ & $0.114(3)$ & $0.022(2)$ & $0.018(2)$ & $0.010(2)$ \\
N1 & $0.0370(12)$ & $0.0694(15)$ & $0.0555(13)$ & $0.0035(10)$ & $0.0089(10)$ & $-0.0248(11)$ \\
N2 & $0.0361(11)$ & $0.0558(13)$ & $0.0544(13)$ & $0.0033(9)$ & $0.0071(9)$ & $-0.0145(10)$ \\
O1 & $0.0688(12)$ & $0.0634(12)$ & $0.0642(12)$ & $0.0126(10)$ & $0.0262(10)$ & $0.0144(10)$ \\
O2 & $0.0382(9)$ & $0.0772(13)$ & $0.0585(11)$ & $0.0056(9)$ & $0.0038(8)$ & $-0.0159(10)$ \\
S1 & $0.0428(3)$ & $0.0552(4)$ & $0.0435(4)$ & $0.0046(3)$ & $0.0095(3)$ & $-0.0070(3)$ \\
C11 & $0.0827(7)$ & $0.1092(8)$ & $0.0920(7)$ & $0.0402(6)$ & $0.0573(6)$ & $0.0216(6)$ \\
C11 & $0.044(3)$ & $0.065(4)$ & $0.067(4)$ & $-0.011(3)$ & $0.001(2)$ & $-0.003(3)$ \\
& & & & & \\
\hline
\end{tabular}

Geometric parameters $\left(\AA,{ }^{\circ}\right)$

\begin{tabular}{llll}
\hline $\mathrm{C} 1-\mathrm{C} 2$ & $1.375(4)$ & $\mathrm{C} 12-\mathrm{C} 13$ & $1.364(4)$ \\
$\mathrm{C} 1-\mathrm{C} 6$ & $1.378(3)$ & $\mathrm{C} 12-\mathrm{H} 12$ & 0.9300 \\
$\mathrm{C} 1-\mathrm{H} 1$ & 0.9300 & $\mathrm{C} 13-\mathrm{H} 13$ & 0.9300 \\
$\mathrm{C} 2-\mathrm{C} 3$ & $1.374(4)$ & $\mathrm{C} 14-\mathrm{C} 15$ & $1.382(3)$ \\
$\mathrm{C} 2-\mathrm{H} 2$ & 0.9300 & $\mathrm{C} 14-\mathrm{C} 19$ & $1.385(3)$ \\
$\mathrm{C} 3-\mathrm{C} 4$ & $1.373(4)$ & $\mathrm{C} 15-\mathrm{C} 16$ & $1.374(4)$ \\
$\mathrm{C} 3-\mathrm{C} 20$ & $1.510(4)$ & $\mathrm{C} 15-\mathrm{H} 15$ & 0.9300 \\
$\mathrm{C} 4-\mathrm{C} 5$ & $1.376(4)$ & $\mathrm{C} 16-\mathrm{C} 17$ & $1.366(4)$ \\
$\mathrm{C} 4-\mathrm{H} 4$ & 0.9300 & $\mathrm{C} 16-\mathrm{H} 16$ & 0.9300 \\
$\mathrm{C} 5-\mathrm{C} 6$ & $1.377(3)$ & $\mathrm{C} 17-\mathrm{C} 18$ & $1.363(4)$ \\
$\mathrm{C} 5-\mathrm{H} 5$ & 0.9300 & $\mathrm{C} 17-\mathrm{C} 11$ & $1.570(5)$ \\
$\mathrm{C} 6-\mathrm{S} 1$ & $\mathrm{C} 17-\mathrm{H} 17$ & $0.905(19)$ \\
$\mathrm{C} 7-\mathrm{N} 2$ & $1.750(2)$ & $\mathrm{C} 18-\mathrm{C} 19$ & $1.378(4)$ \\
$\mathrm{C} 7-\mathrm{C} 14$ & $1.281(3)$ & $\mathrm{C} 18-\mathrm{H} 18$ & 0.9300 \\
$\mathrm{C} 7-\mathrm{C} 8$ & $1.476(3)$ & $\mathrm{C} 19-\mathrm{H} 19$ & 0.9300 \\
$\mathrm{C} 8-\mathrm{C} 13$ & $1.484(3)$ & $\mathrm{C} 20-\mathrm{H} 20 \mathrm{~A}$ & 0.9600 \\
$\mathrm{C} 8-\mathrm{C} 9$ & $1.382(3)$ & $\mathrm{C} 20-\mathrm{H} 20 \mathrm{~B}$ & 0.9600 \\
$\mathrm{C} 9-\mathrm{C} 10$ & $1.383(3)$ & $\mathrm{N} 1-\mathrm{H} 20 \mathrm{C}$ & 0.9600 \\
$\mathrm{C} 9-\mathrm{H} 9$ & $\mathrm{~N} 1-\mathrm{S} 1$ & $1.385(3)$ \\
$\mathrm{C} 10-\mathrm{C} 11$ & $1.382(3)$ & $\mathrm{N} 1-\mathrm{H} 1 \mathrm{~A}$ & $1.639(2)$ \\
$\mathrm{C} 10-\mathrm{H} 10$ & 0.9300 & $\mathrm{O} 1-\mathrm{S} 1$ & $0.877(17)$ \\
$\mathrm{C} 11-\mathrm{C} 12$ & $1.374(4)$ & $\mathrm{O} 2-\mathrm{S} 1$ & $1.4150(18)$ \\
$\mathrm{C} 11-\mathrm{C} 11$ & $\mathrm{C} 8-\mathrm{C} 13-\mathrm{H} 13$ & $1.4296(17)$ \\
$\mathrm{C} 2-\mathrm{C} 1-\mathrm{C} 6$ & 0.9300 & $\mathrm{C} 15-\mathrm{C} 14-\mathrm{C} 19$ & 119.6 \\
$\mathrm{C} 2-\mathrm{C} 1-\mathrm{H} 1$ & $\mathrm{C} 15-\mathrm{C} 14-\mathrm{C} 7$ & $118.3(2)$ \\
$\mathrm{C} 6-\mathrm{C} 1-\mathrm{H} 1$ & $1.365(4)$ & $\mathrm{C} 16-\mathrm{C} 15-\mathrm{C} 14$ & $120.5(2)$ \\
$\mathrm{C} 3-\mathrm{C} 2-\mathrm{C} 1$ & $1.714(3)$ & & $121.2(2)$ \\
$\mathrm{C} 3-\mathrm{C} 2-\mathrm{H} 2$ & $119.4(3)$ & 120.3 & $(3)$
\end{tabular}




\begin{tabular}{|c|c|c|c|}
\hline $\mathrm{C} 1-\mathrm{C} 2-\mathrm{H} 2$ & 119.3 & $\mathrm{C} 16-\mathrm{C} 15-\mathrm{H} 15$ & 119.5 \\
\hline $\mathrm{C} 4-\mathrm{C} 3-\mathrm{C} 2$ & $118.1(3)$ & $\mathrm{C} 14-\mathrm{C} 15-\mathrm{H} 15$ & 119.5 \\
\hline $\mathrm{C} 4-\mathrm{C} 3-\mathrm{C} 20$ & $120.9(3)$ & $\mathrm{C} 17-\mathrm{C} 16-\mathrm{C} 15$ & $119.5(3)$ \\
\hline $\mathrm{C} 2-\mathrm{C} 3-\mathrm{C} 20$ & $120.9(3)$ & $\mathrm{C} 17-\mathrm{C} 16-\mathrm{H} 16$ & 120.3 \\
\hline $\mathrm{C} 3-\mathrm{C} 4-\mathrm{C} 5$ & $121.8(3)$ & $\mathrm{C} 15-\mathrm{C} 16-\mathrm{H} 16$ & 120.3 \\
\hline $\mathrm{C} 3-\mathrm{C} 4-\mathrm{H} 4$ & 119.1 & $\mathrm{C} 18-\mathrm{C} 17-\mathrm{C} 16$ & $121.1(3)$ \\
\hline $\mathrm{C} 5-\mathrm{C} 4-\mathrm{H} 4$ & 119.1 & $\mathrm{C} 18-\mathrm{C} 17-\mathrm{C} 11^{\prime}$ & $115.9(3)$ \\
\hline $\mathrm{C} 4-\mathrm{C} 5-\mathrm{C} 6$ & $119.0(2)$ & $\mathrm{C} 16-\mathrm{C} 17-\mathrm{C} 11^{\prime}$ & $123.1(3)$ \\
\hline $\mathrm{C} 4-\mathrm{C} 5-\mathrm{H} 5$ & 120.5 & $\mathrm{C} 18-\mathrm{C} 17-\mathrm{H} 17$ & $118(3)$ \\
\hline $\mathrm{C} 6-\mathrm{C} 5-\mathrm{H} 5$ & 120.5 & $\mathrm{C} 16-\mathrm{C} 17-\mathrm{H} 17$ & $121(2)$ \\
\hline $\mathrm{C} 5-\mathrm{C} 6-\mathrm{C} 1$ & $120.2(2)$ & $\mathrm{C} 17-\mathrm{C} 18-\mathrm{C} 19$ & $119.5(3)$ \\
\hline $\mathrm{C} 5-\mathrm{C} 6-\mathrm{S} 1$ & $119.72(19)$ & $\mathrm{C} 17-\mathrm{C} 18-\mathrm{H} 18$ & 120.3 \\
\hline $\mathrm{C} 1-\mathrm{C} 6-\mathrm{S} 1$ & $120.04(19)$ & $\mathrm{C} 19-\mathrm{C} 18-\mathrm{H} 18$ & 120.3 \\
\hline $\mathrm{N} 2-\mathrm{C} 7-\mathrm{C} 14$ & $116.3(2)$ & $\mathrm{C} 18-\mathrm{C} 19-\mathrm{C} 14$ & $120.8(3)$ \\
\hline $\mathrm{N} 2-\mathrm{C} 7-\mathrm{C} 8$ & $122.9(2)$ & $\mathrm{C} 18-\mathrm{C} 19-\mathrm{H} 19$ & 119.6 \\
\hline $\mathrm{C} 14-\mathrm{C} 7-\mathrm{C} 8$ & $120.7(2)$ & $\mathrm{C} 14-\mathrm{C} 19-\mathrm{H} 19$ & 119.6 \\
\hline $\mathrm{C} 13-\mathrm{C} 8-\mathrm{C} 9$ & $118.9(2)$ & $\mathrm{C} 3-\mathrm{C} 20-\mathrm{H} 20 \mathrm{~A}$ & 109.5 \\
\hline $\mathrm{C} 13-\mathrm{C} 8-\mathrm{C} 7$ & $119.1(2)$ & $\mathrm{C} 3-\mathrm{C} 20-\mathrm{H} 20 \mathrm{~B}$ & 109.5 \\
\hline $\mathrm{C} 9-\mathrm{C} 8-\mathrm{C} 7$ & $122.0(2)$ & $\mathrm{H} 20 \mathrm{~A}-\mathrm{C} 20-\mathrm{H} 20 \mathrm{~B}$ & 109.5 \\
\hline $\mathrm{C} 10-\mathrm{C} 9-\mathrm{C} 8$ & $120.6(2)$ & $\mathrm{C} 3-\mathrm{C} 20-\mathrm{H} 20 \mathrm{C}$ & 109.5 \\
\hline $\mathrm{C} 10-\mathrm{C} 9-\mathrm{H} 9$ & 119.7 & $\mathrm{H} 20 \mathrm{~A}-\mathrm{C} 20-\mathrm{H} 20 \mathrm{C}$ & 109.5 \\
\hline $\mathrm{C} 8-\mathrm{C} 9-\mathrm{H} 9$ & 119.7 & $\mathrm{H} 20 \mathrm{~B}-\mathrm{C} 20-\mathrm{H} 20 \mathrm{C}$ & 109.5 \\
\hline $\mathrm{C} 11-\mathrm{C} 10-\mathrm{C} 9$ & $118.4(3)$ & $\mathrm{N} 2-\mathrm{N} 1-\mathrm{S} 1$ & $113.42(16)$ \\
\hline $\mathrm{C} 11-\mathrm{C} 10-\mathrm{H} 10$ & 120.8 & $\mathrm{~N} 2-\mathrm{N} 1-\mathrm{H} 1 \mathrm{~A}$ & $121.1(18)$ \\
\hline $\mathrm{C} 9-\mathrm{C} 10-\mathrm{H} 10$ & 120.8 & $\mathrm{~S} 1-\mathrm{N} 1-\mathrm{H} 1 \mathrm{~A}$ & $115.2(17)$ \\
\hline $\mathrm{C} 12-\mathrm{C} 11-\mathrm{C} 10$ & $121.8(2)$ & $\mathrm{C} 7-\mathrm{N} 2-\mathrm{N} 1$ & $117.89(19)$ \\
\hline $\mathrm{C} 12-\mathrm{C} 11-\mathrm{C} 11$ & $118.3(2)$ & $\mathrm{O} 1-\mathrm{S} 1-\mathrm{O} 2$ & $119.43(12)$ \\
\hline $\mathrm{C} 10-\mathrm{C} 11-\mathrm{C} 11$ & $119.9(2)$ & $\mathrm{O} 1-\mathrm{S} 1-\mathrm{N} 1$ & $112.27(12)$ \\
\hline $\mathrm{C} 13-\mathrm{C} 12-\mathrm{C} 11$ & $119.3(3)$ & $\mathrm{O} 2-\mathrm{S} 1-\mathrm{N} 1$ & $103.31(11)$ \\
\hline $\mathrm{C} 13-\mathrm{C} 12-\mathrm{H} 12$ & 120.4 & $\mathrm{O} 1-\mathrm{S} 1-\mathrm{C} 6$ & $107.96(11)$ \\
\hline $\mathrm{C} 11-\mathrm{C} 12-\mathrm{H} 12$ & 120.4 & $\mathrm{O} 2-\mathrm{S} 1-\mathrm{C} 6$ & $110.15(12)$ \\
\hline $\mathrm{C} 12-\mathrm{C} 13-\mathrm{C} 8$ & $120.9(3)$ & $\mathrm{N} 1-\mathrm{S} 1-\mathrm{C} 6$ & $102.39(11)$ \\
\hline $\mathrm{C} 12-\mathrm{C} 13-\mathrm{H} 13$ & 119.6 & & \\
\hline $\mathrm{C} 6-\mathrm{C} 1-\mathrm{C} 2-\mathrm{C} 3$ & $-0.4(4)$ & $\mathrm{C} 8-\mathrm{C} 7-\mathrm{C} 14-\mathrm{C} 15$ & $-176.8(2)$ \\
\hline $\mathrm{C} 1-\mathrm{C} 2-\mathrm{C} 3-\mathrm{C} 4$ & $0.9(4)$ & $\mathrm{N} 2-\mathrm{C} 7-\mathrm{C} 14-\mathrm{C} 19$ & $-175.2(2)$ \\
\hline $\mathrm{C} 1-\mathrm{C} 2-\mathrm{C} 3-\mathrm{C} 20$ & $-178.5(3)$ & $\mathrm{C} 8-\mathrm{C} 7-\mathrm{C} 14-\mathrm{C} 19$ & $3.6(4)$ \\
\hline $\mathrm{C} 2-\mathrm{C} 3-\mathrm{C} 4-\mathrm{C} 5$ & $-0.5(4)$ & $\mathrm{C} 19-\mathrm{C} 14-\mathrm{C} 15-\mathrm{C} 16$ & $0.1(4)$ \\
\hline $\mathrm{C} 20-\mathrm{C} 3-\mathrm{C} 4-\mathrm{C} 5$ & $179.0(3)$ & $\mathrm{C} 7-\mathrm{C} 14-\mathrm{C} 15-\mathrm{C} 16$ & $-179.6(2)$ \\
\hline $\mathrm{C} 3-\mathrm{C} 4-\mathrm{C} 5-\mathrm{C} 6$ & $-0.5(4)$ & $\mathrm{C} 14-\mathrm{C} 15-\mathrm{C} 16-\mathrm{C} 17$ & $-0.5(4)$ \\
\hline $\mathrm{C} 4-\mathrm{C} 5-\mathrm{C} 6-\mathrm{C} 1$ & $1.1(4)$ & $\mathrm{C} 15-\mathrm{C} 16-\mathrm{C} 17-\mathrm{C} 18$ & $0.4(5)$ \\
\hline $\mathrm{C} 4-\mathrm{C} 5-\mathrm{C} 6-\mathrm{S} 1$ & $-178.9(2)$ & $\mathrm{C} 15-\mathrm{C} 16-\mathrm{C} 17-\mathrm{Cl1}^{\prime}$ & $179.3(3)$ \\
\hline $\mathrm{C} 2-\mathrm{C} 1-\mathrm{C} 6-\mathrm{C} 5$ & $-0.7(4)$ & $\mathrm{C} 16-\mathrm{C} 17-\mathrm{C} 18-\mathrm{C} 19$ & $0.2(5)$ \\
\hline $\mathrm{C} 2-\mathrm{C} 1-\mathrm{C} 6-\mathrm{S} 1$ & $179.3(2)$ & $\mathrm{C} 11^{\prime}-\mathrm{C} 17-\mathrm{C} 18-\mathrm{C} 19$ & $-178.8(3)$ \\
\hline $\mathrm{N} 2-\mathrm{C} 7-\mathrm{C} 8-\mathrm{C} 13$ & $64.3(3)$ & $\mathrm{C} 17-\mathrm{C} 18-\mathrm{C} 19-\mathrm{C} 14$ & $-0.6(5)$ \\
\hline $\mathrm{C} 14-\mathrm{C} 7-\mathrm{C} 8-\mathrm{C} 13$ & $-114.3(3)$ & $\mathrm{C} 15-\mathrm{C} 14-\mathrm{C} 19-\mathrm{C} 18$ & $0.5(4)$ \\
\hline $\mathrm{N} 2-\mathrm{C} 7-\mathrm{C} 8-\mathrm{C} 9$ & $-115.0(3)$ & $\mathrm{C} 7-\mathrm{C} 14-\mathrm{C} 19-\mathrm{C} 18$ & $-179.8(3)$ \\
\hline $\mathrm{C} 14-\mathrm{C} 7-\mathrm{C} 8-\mathrm{C} 9$ & $66.4(3)$ & $\mathrm{C} 14-\mathrm{C} 7-\mathrm{N} 2-\mathrm{N} 1$ & $178.9(2)$ \\
\hline
\end{tabular}




\begin{tabular}{llll}
$\mathrm{C} 13-\mathrm{C} 8-\mathrm{C} 9-\mathrm{C} 10$ & $-0.8(4)$ & $\mathrm{C} 8-\mathrm{C} 7-\mathrm{N} 2-\mathrm{N} 1$ & $0.2(4)$ \\
$\mathrm{C} 7-\mathrm{C} 8-\mathrm{C} 9-\mathrm{C} 10$ & $178.5(2)$ & $\mathrm{S} 1-\mathrm{N} 1-\mathrm{N} 2-\mathrm{C} 7$ & $168.96(19)$ \\
$\mathrm{C} 8-\mathrm{C} 9-\mathrm{C} 10-\mathrm{C} 11$ & $0.1(4)$ & $\mathrm{N} 2-\mathrm{N} 1-\mathrm{S} 1-\mathrm{O} 1$ & $-50.0(2)$ \\
$\mathrm{C} 9-\mathrm{C} 10-\mathrm{C} 11-\mathrm{C} 12$ & $0.4(4)$ & $\mathrm{N} 2-\mathrm{N} 1-\mathrm{S} 1-\mathrm{O} 2$ & $-179.99(18)$ \\
$\mathrm{C} 9-\mathrm{C} 10-\mathrm{C} 11-\mathrm{C} 11$ & $179.4(2)$ & $\mathrm{N} 2-\mathrm{N} 1-\mathrm{S} 1-\mathrm{C} 6$ & $65.5(2)$ \\
$\mathrm{C} 10-\mathrm{C} 11-\mathrm{C} 12-\mathrm{C} 13$ & $-0.1(4)$ & $\mathrm{C} 5-\mathrm{C} 6-\mathrm{S} 1-\mathrm{O} 1$ & $17.3(2)$ \\
$\mathrm{C} 11-\mathrm{C} 11-\mathrm{C} 12-\mathrm{C} 13$ & $-179.2(2)$ & $\mathrm{C} 1-\mathrm{C} 6-\mathrm{S} 1-\mathrm{O} 1$ & $-162.68(19)$ \\
$\mathrm{C} 11-\mathrm{C} 12-\mathrm{C} 13-\mathrm{C} 8$ & $-0.6(4)$ & $\mathrm{C} 5-\mathrm{C} 6-\mathrm{S} 1-\mathrm{O} 2$ & $149.31(19)$ \\
$\mathrm{C} 9-\mathrm{C} 8-\mathrm{C} 13-\mathrm{C} 12$ & $1.0(4)$ & $\mathrm{C} 1-\mathrm{C} 6-\mathrm{S} 1-\mathrm{O} 2$ & $-30.7(2)$ \\
$\mathrm{C} 7-\mathrm{C} 8-\mathrm{C} 13-\mathrm{C} 12$ & $-178.3(2)$ & $\mathrm{C} 5-\mathrm{C} 6-\mathrm{S} 1-\mathrm{N} 1$ & $-101.3(2)$ \\
$\mathrm{N} 2-\mathrm{C} 7-\mathrm{C} 14-\mathrm{C} 15$ & $4.5(3)$ & $\mathrm{C} 1-\mathrm{C} 6-\mathrm{S} 1-\mathrm{N} 1$ & $78.7(2)$ \\
\hline
\end{tabular}

Hydrogen-bond geometry $\left(\AA,{ }^{\circ}\right)$

\begin{tabular}{lllll}
\hline$D-\mathrm{H} \cdots A$ & $D-\mathrm{H}$ & $\mathrm{H} \cdots A$ & $D \cdots A$ & $D-\mathrm{H} \cdots A$ \\
\hline $\mathrm{N} 1-\mathrm{H} 1 A \cdots \mathrm{O} 2^{\mathrm{i}}$ & $0.88(2)$ & $2.19(2)$ & $3.024(3)$ & $160(2)$ \\
$\mathrm{C} 1-\mathrm{H} 1 \cdots \mathrm{C} 11^{1 i}$ & 0.93 & 2.91 & $3.694(3)$ & 143 \\
$\mathrm{C} 10-\mathrm{H} 10 \cdots \mathrm{Cl1}^{\prime}{ }^{\prime i i}$ & 0.93 & 2.76 & $3.476(7)$ & 134 \\
$\mathrm{C} 16-\mathrm{H} 16 \cdots{ }^{\text {iv }}$ & 0.93 & 2.54 & $3.339(3)$ & 145 \\
\hline
\end{tabular}

Symmetry codes: (i) $-x+1,-y+2,-z+1$; (ii) $-x+1, y-1 / 2,-z+3 / 2$; (iii) $-x+2, y+1 / 2,-z+3 / 2$; (iv) $-x+2,-y+2,-z+1$. 